\title{
Factores Blandos en la Gestión de Integración de las Cadenas y/o Redes de Abastecimiento: Aproximación a un Modelo Conceptual
}

\author{
Juan C. Aldana-Bernal ${ }^{(1)}$ y César A. Bernal-Torres ${ }^{(2)^{*}}$ \\ (1) Universidad Nacional de Colombia, Facultad de Ingeniería, Departamento de Sistemas Industriales, \\ Carrera 45 n. ${ }^{\circ} 26-85$, Edificio Aulas de Ingeniería, Bogotá, Colombia. (e-mail: jcaldanab@unal.edu.co). \\ (2) Universidad de La Sabana, Escuela Internacional de Ciencias Económicas y Administrativas, \\ Km. 7, Autopista Norte de Bogotá, Puente del Común, Campus Universitario, Chía, Colombia. \\ (e-mail: cesar.bernal@unisabana.edu.co). \\ * Autor a quien debe ser dirigida la correspondencia.
}

Recibido Jun. 30, 2017; Aceptado Ago. 30, 2017; Versión final Oct. 30, 2017, Publicado Abr. 2018

\begin{abstract}
Resumen
Este artículo analiza la importancia de los factores blandos en la gestión de integración en las cadenas/ redes de abastecimiento y plantea un modelo conceptual que muestra el rol de esos factores en las cadenas/redes. La revisión de la literatura mostró que los determinantes blandos de la integración y gestión de esas cadenas corresponden a factores intangibles asociados a la forma en que las organizaciones se relacionan y se comportan. También se encontró que, aunque la literatura menciona diversos factores blandos, casi siempre analizados de forma separada por los diferentes autores, todos ellos se pueden agrupar en tres factores: el capital social, la cultura organizacional y la gestión del conocimiento. Estos tres factores integran el modelo propuesto. El Modelo propone que toda cadena/red de abastecimiento requiere ser pensada como una estrategia competitiva. Para ello, es necesario considerar estos factores como relevantes, de forma integrada, porque contribuyen a generar valor de manera sostenible tanto para cada empresa como para el conjunto de las empresas y partes interesadas de la cadena/red.
\end{abstract}

\section{Soft Factors in the Management of Integration Supply Chains and/or Networks: Approximation to a Conceptual Model}

\begin{abstract}
This article analyzes the importance of soft factors in the interaction management in supply chains/networks, and presents a conceptual model that shows the role of these factors in these chains / networks. The review of the literature shows that soft determinants of integration and management of these chains correspond to intangible factors associated with the way organizations relate and behave. It was also found that, although the literature mentions several soft factors, almost always analyzed separately by the different authors, for this study, they are all grouped into three factors: social capital, organizational culture and knowledge management. These three factors are considered in the proposed model. The model suggests that all supply chain/network needs to be considered as a competitive strategy and for this it is necessary to consider these factors as relevant, in an integrated way, because they contribute to generate value in a sustainable way for each company as well as for all the companies and stakeholders of the chain / network.
\end{abstract}

Keywords: supply chains; business integration; soft factors; competitiveness; sustainability 


\section{INTRODUCCIÓN}

Es indiscutible que las organizaciones, para cumplir sus propósitos, son sistemas abiertos con estructuras que implican relaciones con otras organizaciones. Por esto, para mejorar sus desempeño individual, es esencial la coordinación de los procesos de negocio mediante la integración de manera eficiente, eficaz (Mentzer et al., 2001) y sostenible (Laszlo, 2003; OIT, 2012). Por lo tanto, la integración interorganizacional ha de ser considerada como un proceso prioritario de toda organización dado que ella pretende establecer flujos y vínculos de largo plazo entre una organización y otra u otras organizaciones en su entorno. Desde esta perspectiva, la gestión de la cadena de abastecimiento (SCM, por sus siglas en inglés) tiene como fundamento la integración.

Lambert y Cooper (2000) sugieren que la estructura de la SCM surge de la necesidad de integrar los diferentes eslabones dentro de la cadena y/o red de abastecimiento como son los proveedores, fabricantes y clientes. En otras palabras, la integración es identificada como una de las características esenciales en la gestión de la cadena de abastecimiento dado que, usualmente, se la describe como la interacción a través de interfaces normalizadas para reducir el esfuerzo requerido del procesamiento de la información entre los integrantes de la cadena (Mentzer et al., 2001). En este sentido, esta visión se ha venido incrementando con el uso de sistemas colaborativos que permiten la visibilidad de toda la cadena de abastecimiento (Govindan et al., 2013; Tseng et al., 2015). Hay, en la actualidad, una tendencia a considerar la cadena de abastecimiento, no solamente desde el ámbito diádico de cliente-proveedor sino bajo una perspectiva de red de abastecimiento, como aquella en la cual las organizaciones están vinculadas con diferentes socios de negocios de su sector en los procesos fundamentales de estas organizaciones, para la generación de valor en los productos y servicios proporcionados al cliente final a través de estas actividades (Christopher, 1998).

En este sentido, la integración en la cadena de abastecimiento es definida como la forma en que se pueden soportar los procesos de negocio de una organización a través de la red de abastecimiento (Romano, 2003) y que, por lo mismo, debe involucrar la integración interna con las organizaciones proveedoras de suministros y demandantes de bienes o servicios (Vickery et al., 2004) con el propósito de lograr flujos eficientes de productos, servicios, información y decisiones que proporcionen valor al cliente (Flynn et al., 2010) e impacten positivamente el desempeño de las organizaciones (Aryee et al., 2008) en sus diferentes grupos de interés (Fontrodona y Sison, 2006). También es entendida como el grado en el cual tres o más organizaciones, con alguna forma de relación sectorial, soportadas en la confianza recíproca y la identificación de objetivos comunes a largo plazo, establecen acuerdos para consolidar sus procesos de interacción mutua mediante el intercambio de información, recursos, tecnologías y compartición de riesgos y beneficios que, en su conjunto, les permiten un mejor desempeño y el desarrollo de ventajas competitivas que no podrían ser alcanzadas por cada empresa individualmente (Borgatti y Li, 2009).

A este respecto y dado que la integración en la cadena de abastecimiento es identificada como un nivel de interacción, usualmente se la asocia a tres instancias: la primera es la integración interna, que se constituye en el fundamento para la segunda, que es la integración con proveedores, la cual, a su vez, es el requisito para la tercera instancia: la integración con clientes y demás grupos de interés externos. Así, la integración interna es el grado en el cual una empresa estructura sus propias estrategias, prácticas y procesos mediante sistemas colaborativos y sincronizados para cumplir eficazmente los requerimientos del cliente y de sus grupos de interés (Flynn et al., 2010). Para estos autores, la integración con proveedores se entiende en los mismos términos de la integración interna, con la diferencia que involucra el desarrollo de competencias fundamentales con proveedores críticos. Finalmente, la integración con el cliente se entiende bajo los mismos conceptos pero con sus clientes externos y grupos de interés mediante sistemas colaborativos y sincronizados.

De otra parte, la revisión de la literatura indica que la gestión de las cadenas de abastecimiento han sido frecuentemente estudiadas desde la integración de los factores técnicos (métodos de planeación y control, estructura organizacional, cercanía física y compartición de activos y de flujos de actividades, información y productos), mediante un enfoque que no ha podido explicar, de manera completa y satisfactoria, la dinámica de las cadenas de abastecimiento debido, en gran parte, a la no inclusión de los factores soft o blandos que son determinantes en la integración de toda la cadena (Naslund y Hulthen, 2012). Por esta razón, surge la necesidad de profundizar en el estudio de los factores soft en la integración de la cadena de abastecimiento para su mejor comprensión.

Ante esta perspectiva, el presente artículo se orientó a revisar la literatura sobre los factores blandos en la integración de la cadena de abastecimiento y, en consecuencia, a proponer un modelo conceptual de relaciones en esa cadena, en la que los factores blandos juegan un papel importante, a aportar evidencia que contribuya a la comprensión del fundamento de las cadenas de abastecimiento y a que los responsables de la gestión de esa cadena logren una mayor eficacia en la creación de valor para las empresas y el conjunto 
de sus grupos de interés por cuanto existe evidencia de que la integración empresarial en las cadenas de abastecimiento tiene un marcado impacto positivo en el desempeño y la competitividad organizacional (Turgay y Ekemen, 2013).

Esta investigación, de tipo documental, se fundamentó en la revisión de la bibliografía publicada en las bases de datos ISI Web, Scopus y Science Direct referida a las siguientes temáticas: investigaciones asociadas a la integración interempresarial, integración en la administración de la cadena de abastecimiento (SCM) e investigaciones relacionadas con determinantes o factores que impactan dicha integración interempresarial y la SCM y factores blandos (soft) o intangibles que impactan la integración en redes y cadenas de abastecimiento.

De la revisión de la literatura se encontró que, sobre el tema, se mencionan una diversidad de factores blandos determinantes de la eficacia de la cadena de abastecimiento y que, dependiendo del enfoque que cada estudioso da a su investigación, se enfatiza en alguno o varios de esos factores, pero ningún autor revisado integra el conjunto de estos. De otra parte, se encontró que la diversidad de factores blandos citados se puede sintetizar en sólo tres: el capital social, la cultura organizacional y la gestión del conocimiento, porque los demás (la confianza, la cooperación, el poder y el riesgo, el compromiso y la orientación al mercado) son, en realidad, rasgos de alguno de estos tres factores mencionados y no factores considerados como tales. El objetivo de la integración del conjunto de los factores en los tres mencionados es evitar la confusión de significados que genera la actual diversidad de factores sugeridos por los autores consultados dado que algunos de los considerados factores, como se acaba de indicar, son rasgos y no propiamente factores.

El artículo está estructurado en las siguientes partes: la introducción, que sintetiza el contenido del artículo; la revisión de la literatura, que muestra la importancia de la integración de la cadena de abastecimiento para las organizaciones de toda índole, los factores limitantes de esa integración y la identificación de los factores blandos y su rol en esa integración; la metodología utilizada para el desarrollo del estudio que indica el proceso seguido para la estructuración del modelo; el modelo propuesto con la descripción de sus características; y la discusión de los resultados y las conclusiones a las que se llegó.

\section{FACTORES DE INTEGRACIÓN EN LA SCM}

En lo referente a la gestión de la cadena de abastecimiento es usual una pregunta: ¿Qué motiva a las organizaciones a integrarse en su cadena de abastecimiento? Esta pregunta es respondida por Lambert y Cooper (2000) al definir la cadena de abastecimiento como un sistema compuesto por tres elementos fundamentales que están interrelacionados: el primero es la estructura de la red de la cadena de abastecimiento, definida por las empresas y la forma como estas se relacionan; el segundo son los procesos de negocio de la cadena de abastecimiento que definen cómo esta cadena agrega valor al cliente final y a los demás grupos de interés de las empresas de esa cadena mediante la mejora en la cooperación, los sistemas de gestión y las medidas de desempeño tales como la reducción de costos (Sanchis et al., 2009); y el tercero es el componente gerencial que define la forma como los procesos de negocio se integran a través de la cadena.

En el tercer elemento se identifican los componentes motivadores que determinan el proceso de integración, los cuales se dividen en dos: (a) los componentes físicos y técnicos de la cadena, correspondientes a aspectos tangibles o duros, y (b) los componentes gerenciales y de comportamiento de la cadena, que corresponden a los aspectos intangibles o blandos. Los componentes físicos y técnicos, que corresponden a la primera división, son caracterizados por ser tangibles y fácilmente medibles, por lo cual son identificados como aspectos duros y, usualmente, son referidos a aspectos tales como los métodos de planeación y control, la estructura organizacional, la cercanía física y la compartición de activos y de flujos de actividades, información y productos. Desde esta perspectiva, los componentes gerenciales y de comportamiento son considerados esenciales porque determinan la forma como los componentes duros (técnicos) pueden ser implementados por cuanto, de no estar adecuadamente alineados, pueden hacer menos eficaz la cadena y menos competitivas a cada una de las organizaciones de esa cadena (Lambert y Cooper, 2000).

Como consecuencia de lo antes mencionado, el estudio de los determinantes técnicos no ha podido explicar de manera completa y satisfactoria la dinámica de las cadenas de abastecimiento. Así lo evidencia la revisión bibliográfica que realizaron Naslund y Hulthen (2012) al encontrar en los estudios de eficacia de las cadenas de abastecimiento que sólo se han considerado, frecuentemente, los factores técnicos (duros) de la integración en la SCM y se han desconocido los factores soft o blandos que determinan la integración. Por lo tanto, los mencionados autores sugieren profundizar en el estudio de los factores soft de la integración en la cadena de abastecimiento para una mejor comprensión de esa integración. Ante esta perspectiva, el presente artículo se orientó a revisar la literatura sobre el rol de los factores blandos en la integración de la cadena de abastecimiento y, en consecuencia, a proponer un modelo conceptual de relaciones en esa cadena en la que 
los factores blandos juegan un papel importante y a aportar evidencia que contribuya a la comprensión del fundamento de las cadenas de abastecimiento y a que los responsables de la gestión de esa cadena logren una mayor eficacia en la creación de valor para las empresas y el conjunto de sus grupos de interés.

\section{Factores limitantes para la integración en la SCM}

En la actualidad se han identificado algunos factores que están asociados a las barreras que limitan la adecuada integración de las empresas en sus cadenas de abastecimiento que impiden alcanzar los objetivos esperados en la mejora de la competitividad en las empresas involucradas (Wu et al., 2004). Estas diferentes barreras, que obstaculizan la integración de las empresas en las cadenas de abastecimiento, fueron estudiadas por Katunzi (2011), quien identificó varios aspectos como los posibles obstáculos para la integración, entre los cuales se mencionan: la mentalidad de silo, es decir, no considerar el impacto de sus acciones sobre los otros; la falta de visibilidad en la cadena de suministro, o, lo que es lo mismo, no trabajar de manera coordinada; la falta de confianza que se produce cuando no se crea una buena imagen y reputación entre los miembros de la cadena; la falta de conocimiento, que evidencia que las empresas no comparten ni colaboran con sus pares en la cadena; y el nivel diádico de las relaciones, es decir, que las relaciones sólo se estructuran en parejas proveedor-cliente.

De acuerdo con Katunzi (2011), el foco de los problemas de la integración en la SCM hace referencia a los factores blandos y comportamentales de la integración. Por esta razón, es necesario profundizar en su estudio y análisis porque, al ser entendidos y desarrollados de manera adecuada, se pueden dar luces a la solución de los problemas que hasta ahora no han tenido una solución apropiada. A este respecto, Naslund y Hulthen (2012), luego de una amplia revisión de la literatura acerca de la integración en la SCM, encontraron dos problemas principales para la integración en la SCM. El primero es que la integración en la cadena de abastecimiento se ha desarrollado, empíricamente, en un nivel diádico (cliente-proveedor) y no encuentran muchas evidencias de cadenas de abastecimiento integradas desde el proveedor primario hasta el cliente final en una estructura de red. Esta consideración puede ser entendida como una consecuencia de las dificultades planteadas por Katunzi (2011) en la medida en que la falta de confianza y visión de grupo hace que las empresas establezcan acuerdos muy limitados en la cadena. El segundo problema hace referencia a la falta de homologación en la literatura acerca del concepto de integración en la cadena de abastecimiento y su diferencia con el término de colaboración, que no ha tenido un lineamiento común.

\section{Factores blandos de la integración}

Los factores blandos en la integración en la SCM son definidos como los factores gerenciales y comportamentales de esa integración (Lambert y Cooper, 2000) que, usualmente, no son tangibles y, por lo tanto, son más complejos de medir y de cambiar que los factores técnicos. Si estos factores no están alineados con los objetivos y las operaciones de la cadena de abastecimiento, en su conjunto, la empresa y su cadena de abastecimiento es menos eficaz en su desempeño. Por este motivo, es importante analizar los aspectos blandos más relevantes que, como se ha mencionado, están relacionados con los obstáculos de la integración en la SCM y son el fundamento de la conceptualización de esa integración en la SCM bajo la perspectiva de red.

De acuerdo con la revisión de la literatura realizada para este artículo, se identificó que los factores blandos más recurrentes, 2004a en los procesos de integración son los siguientes: el capital social (Carey et al., 2011; Min et al., 2008; Putnam, 2004; Roden y Lawson, 2014; Villena et al., 2011; Wu et al., 2004), la identificación de metas y propósitos comunes (Assif , 2011; Mentzer et al., 2001; Min et al., 2008), la confianza (Assif, 2011), la cultura organizacional (AISagheer et al., 2011; Braunscheidel et al., 2010; Lambert y Cooper, 2000; Naslund y Hulthen, 2012), la gestión del conocimiento (Govindan et al., 2013; López et al., 2014; Mentzer et al., 2001; Naslund y Hulthen, 2012; Saraf et al., 2007; Simanca et al., 2015; Tseng et al., 2015; Von Hippel, 1998), la cooperación (Aryee et al., 2008; Mentzer et al., 2001; Naslund y Hulthen, 2012; Mohr et al., 1996), el poder y el riesgo compartido (Assif , 2011; Lambert y Cooper, 2000; Mentzer et al., 2001), el compromiso (Anderson y Weitz, 1992; Sukati et al., 2012), la orientación al mercado (Agan, 2011; Connell y Voola, 2013). La siguiente es una presentación de cada uno de estos factores:

El capital social, entendido como "el conjunto de relaciones e interacciones entre los diferentes actores y los procesos derivados de estas interacciones en la cadena de abastecimiento" (Min et al., 2008, p. 4). Este factor está constituido por el capital estructural que hace referencia al número de relaciones de parentesco, amistad o confianza con los proveedores, clientes y competidores, así como a la posición que la empresa ocupa en el sistema de red, para determinar su accesibilidad para cualquier otra empresa con relaciones de largo plazo (Borgatti y Li, 2009; Lee, 2005); el capital cognitivo, que hace relación a las metas compartidas y al grado en que los miembros de la red comparten el entendimiento y una aproximación a las realizaciones comunes y que busca el desarrollo de políticas que eviten la redundancia y permitan la mayor efectividad en las 
realizaciones con máximo beneficio para las partes (Assif, 2011; Mentzer et al., 2001); el capital relacional, definido como el rol de cada actor en una relación y de cómo se deben alcanzar los resultados esperados (Inkpen et al., 2005; Roden y Lawson, 2014), está asociado con la confianza junto con la identificación y la obligación entre los actores de una relación (Assif, 2011). Diferentes estudios han encontrado una asociación positiva entre el desarrollo del capital social y la estrechez en las relaciones de las empresas en la cadena de abastecimiento (Horn et al., 2014; Son et al., 2016; Oliveira, 2013; Min et al., 2008; Roden y Lawson, 2014; Villena et al., 2011; Carey et al., 2011; Lawson, 2008).

La identificación de metas y propósitos comunes entre los miembros de la red y/o cadena (Min et al., 2008) está relacionado con el desarrollo del capital cognitivo, mediante la construcción de una cultura centrada en unos valores compartidos con sus socios, en la red de abastecimiento, en donde se puedan establecer metas comunes mediante la definición de políticas que permitan alcanzar los proyectos conjuntos de la forma más eficaz, eficiente y sostenible (Assif, 2011; Mentzer et al., 2001).

La confianza, definida como la base en la cual se soportan las relaciones en la red y/o cadena de abastecimiento, se encuentra asociada al capital relacional. Este es un factor primordial porque fomenta y perpetúa las relaciones sociales exitosas de la red interempresarial (Dwyer y Singh, 1998). La confianza, igualmente, está asociada a las expectativas, suposiciones o creencias que un miembro de la SCM tiene sobre la posibilidad de que las acciones futuras de los otros miembros de la cadena serán beneficiosas, favorables o, al menos, no perjudiciales. Algunos aspectos que son identificados como significativos en el desarrollo de la confianza en los procesos de integración son la velocidad de la integración, la calidad de la comunicación y el multiculturalismo adquirente. De igual manera, hay estudios en la literatura que identifican el nivel de confianza de una parte sobre la otra mediante la percepción de competencia, benevolencia, integridad, transparencia y el valor de congruencia (Assif, 2011).

La cultura organizacional es entendida como el grado en el que los valores y las normas de comportamiento compartido dirigen las relaciones de una organización (AISagheer et al., 2011; Lambert y Cooper, 2000; Naslund y Hulthen, 2012). Este es un factor blando significativo por cuanto, según Braunscheidel et al. (2010), tiene un gran impacto sobre las prácticas de integración en la SCM. La cultura y el lenguaje son necesarios para compartir interpretaciones que tienen las empresas sobre su gestión y están asociados al capital cognitivo (Inkpen et al., 2005). Específicamente se evidencia que la cultura jerárquica tiene un impacto negativo en la integración interna y externa mientras que la cultura adhocrática tiene un impacto positivo en la integración externa, lo cual puede deberse a que este tipo de cultura fomenta el emprendimiento y la creación de nuevo conocimiento (Braunscheidel et al., 2010).

En la cultura organizacional, la comunicación colaborativa es un factor fundamental pues es entendida como las actitudes y los procesos colaborativos de los miembros de la cadena para guiar y administrar las relaciones de la cadena en un ambiente de apoyo mutuo y respeto (Mohr et al., 1996). Si la comunicación colaborativa se fundamenta en intereses compartidos y objetivos comunes, puede lograr el desarrollo de otro factor como es el compromiso incremental de los integrantes de la cadena. La comunicación colaborativa puede ser medida en diferentes dimensiones, algunas de ellas son: la frecuencia, que hace referencia a la cantidad de contactos que establecen los miembros de la cadena; la bidireccionalidad, entendida como el grado en que se envían y reciben comunicaciones verticales en las jerarquías de las organizaciones; y la formalidad, que hace referencia a la estructura, planeación y medio de la comunicación (Mohr et al., 1996). El que sea no coercitivo se refiere a la no utilización de alguna influencia impositiva para cambiar comportamientos de otros miembros de la cadena. Así mismo, otras investigaciones sugieren que el grado de comunicación y la confianza entre los administradores de las organizaciones con sus pares influyen en la integración de la cadena de abastecimiento (Lee, 2005).

La gestión del conocimiento entre los miembros de la red y/o cadena es visualizada como un requisito para desarrollar una filosofía de integración en la SCM, especialmente para los procesos de planeación y control (Simanca et al., 2015; López et al., 2014; Mentzer et al., 2001; Naslund y Hulthen, 2012). El compartir información y crear y usar conocimiento se define como la voluntad de los miembros de la cadena de hacer disponible el conocimiento estratégico y táctico a otros miembros de la cadena para la creación de mecanismos de interacción entre las empresas y permitir así la transferencia, recombinación o creación de conocimiento especializado (Govindan et al., 2013; Tseng et al., 2015). Según Saraf et al. (2007), hay dos tipos de activos relacionales que se asocian significativamente con el resultado del proceso de integración: el compartir conocimiento de negocios con el canal de los socios y el acoplamiento al proceso con los clientes. Para Capo-Vicedo et al. (2007), una cadena de abastecimiento debe estructurarse como una red en donde se eliminen las barreras al desarrollo, gestión y transmisión del conocimiento entre sus miembros. En este mismo sentido, Von Hippel (1998) propone que si en un sistema de red se cuenta con mecanismos de transferencia de conocimientos muy bien desarrollados entre los diferentes usuarios, tales como los proveedores y fabricantes, ellos serán capaces de generar innovación de producción en redes con 
compartición de conocimiento. Estos estudios sugieren que los socios aliados de una empresa son, en muchos casos, la más importante fuente de nuevas ideas e información que generan nuevas tecnologías e innovaciones que mejoran el rendimiento.

La cooperación es entendida como el conjunto de actividades coordinadas que desarrollan las empresas de la SCM en las relaciones de negocios para generar resultados superiores beneficiando a todos los miembros, que, individualmente, no podrían ser alcanzados por cada empresa (Mentzer et al., 2001). La cooperación no está limitada a los diferentes niveles jerárquicos de una organización sino que es una coordinación transversal de todos los diferentes miembros de la cadena. La cooperación también es concebida como una estrategia organizacional que involucra el poder, la estructura, los acuerdos, la confianza y el riesgo compartido entre los miembros de la cadena (Aryee et al., 2008; Naslund y Hulthen, 2012). El desarrollo de este factor podría minimizar tres de los obstáculos identificados para la integración al permitir a una organización no tener mentalidad de silo, alcanzar más visibilidad en la cadena y lograr más colaboración con los miembros de la cadena para ser más eficientes, eficaces y sostenibles.

El poder y el riesgo compartido, donde el poder es definido como la capacidad de liderazgo, es decir, la habilidad que tienen algunas empresas para influir en otras empresas de su red y/o cadena de abastecimiento en el desarrollo de alguna acción y así desarrollar la capacidad de generar compromiso (Assif , 2011; Lambert y Cooper, 2000), mientras que, por su parte, el riesgo compartido hace referencia a la forma como las organizaciones de la cadena están dispuestas a comprometerse para participar de los beneficios y de los aspectos negativos que genere la relación en el largo plazo (Lambert y Cooper, 2000; Mentzer et al., 2001).

El compromiso, definido como el deseo de continuar la relación y asegurar su permanencia, se constituye en una promesa por parte de los miembros de la cadena para dar continuidad a la relación de integración y generarle estabilidad (Anderson y Weitz, 1992). En este sentido, se busca que los miembros de la cadena prioricen el establecer y mantener relaciones de largo plazo sobre las de corto plazo de manera que se pueda tener un mayor conocimiento de cada socio y se pueda profundizar más estrechamente en las relaciones.

La orientación al mercado, definida como una cultura organizacional fundamentada en la confianza, la empatía y la comunicación, produce rendimientos si genera un valor superior para los clientes (Agan, 2011; Connell y Voola, 2013) y demás grupos de interés de las empresas de la cadena (Fontrodona et al., 2006). Una métrica identificada para medir esta orientación al mercado define tres componentes: la orientación al cliente, la orientación a la competencia y la coordinación interfuncional (Agan, 2011).

De lo antes expuesto se observa que, en relación con los factores blandos determinantes de la integración interempresarial en la gestión de la cadena de abastecimiento, son diversos los factores que la literatura revisada menciona. También se observa que cada uno de los diferentes estudiosos del tema enfatiza y describe el rol de uno o varios de esos factores en la cadena, pero no el rol del conjunto de estos. Además, al analizar el significado de esos factores se observa que el conjunto de ellos se puede sintetizar en sólo tres: el capital social, la cultura organizacional y la gestión del conocimiento, porque los demás (la identificación de metas y propósitos comunes, la confianza, la cooperación, el poder y el riesgo compartido, el compromiso y la orientación al mercado) en realidad son rasgos de alguno de los tres factores mencionados y no factores definidos como tales; por ejemplo, la confianza, la cooperación (colaboración) y el compromiso son mencionados, entre otros, como rasgos del capital social, la cultura organizacional y la gestión del conocimiento por los diferentes autores de estos tres factores.

Esta diversidad de factores blandos de la SCM y la falta de claridad en su significado obedecen, en gran parte, a los enfoques parciales que han dado los diferentes estudiosos del tema en sus investigaciones y a la poca rigurosidad de los mismos por precisar su significado. Esto tiene el inconveniente de generar una confusión conceptual sobre el significado de los factores en quienes tienen interés por profundizar sobre el tema o en quienes, por diferente motivo, se acercan al conocimiento de estas investigaciones.

\section{METODOLOGÍA}

Esta es una investigación de tipo documental fundamentada en la revisión de la bibliografía publicada en las bases de datos ISI Web, Scopus y ScienceDirect. La búsqueda se inició filtrando, en las bases de datos, conceptos amplios como integración de negocios y aquellos conceptos asociados a investigaciones referentes a integración sectorial (sectoral integration) e integración en la gestión de la cadena de abastecimiento (SCM integration). De esta revisión se encontró que los principales journals que más publican sobre el tema son: Journal of Operations Management, Journal of Production Research, Journal of Management Research, Journal of Business Logistics, International Journal of Physical Distribution \& Logistics Management y Supply Chain Forum An International Journals. La tabla 1 muestra el número de artículos encontrados referentes a integración y gestión de las cadenas/redes de abastecimiento. 
Tabla 1: Relación de artículos consultados sobre integración de la cadena de abastecimiento

\begin{tabular}{|l|l|l|l|l|}
\hline Período/publicación & Business Integration & Sectoral Integration & Supply networks & SCM Integration \\
\hline Antes de 2000 & 16 & 3 & 10 & 8 \\
\hline 2000 - 2009 & 14 & 13 & 15 & 34 \\
\hline Posterior a 2009 & 12 & 11 & 10 & 24 \\
\hline Total & 42 & 27 & 35 & 66 \\
\hline
\end{tabular}

Del conjunto de artículos (66) encontrados sobre la integración de las cadenas de abastecimiento, el análisis se centró en los relacionados con los determinantes para los procesos de integración interempresarial y, en particular, en aquellos que hacían referencia directa a los factores blandos (soft) o intangibles, como determinantes de esos procesos (53 artículos), por ser este el tema central de este artículo. A partir de esa revisión de la literatura encontrada se identificaron los diferentes factores blandos considerados determinantes en la integración de la gestión de la cadena o red de abastecimiento, se hizo la depuración de los mismos y se procedió a estructurar el modelo aquí propuesto.

\section{PROPUESTA DE MODELO}

El modelo de cadena de abastecimiento con énfasis en los factores blandos busca responder a las diferentes inquietudes de los estudios citados en la revisión de la literatura disponible que han evaluado diversas perspectivas acerca de cómo algunos factores blandos tienen impacto positivo en la forma en que las empresas se relacionan e integran en sus cadenas de abastecimiento y, consecuentemente, en la sostenibilidad de esta red de empresas en sus respectivos sectores. El modelo propuesto es un complemento a los modelos que enfatizan los factores técnicos en la gestión de las redes y cadenas de abastecimiento e induce al lector a concebir las cadenas no sólo como unas simples relaciones diádicas entre las partes individuales (cada empresa o actor) de la cadena sino a hacerlo con unas perspectivas de mayor nivel que resalten las interacciones, es decir, los comportamientos sociales de las personas de las empresas más que las empresas en sí; acentúa también sobre los factores blandos de la cadena de abastecimiento como una estrategia de red social que permite armonizar los múltiples (e incluso contradictorios) intereses interdependientes de las partes interesadas; y reconoce que las organizaciones y sus diversas partes interesadas están integradas en redes sociales interconectadas y que sus relaciones entre sí y con la organización son dinámicas y complejas.

El modelo enfatiza en la idea que toda empresa con visión de largo plazo debe concebir a la cadena de abastecimiento como una de sus estrategias competitivas (Turgay y Ekemen, 2013) y, por ende, es parte de su direccionamiento estratégico que contribuye a responder de forma integral no sólo a los requerimientos de los accionistas y los clientes sino a los del conjunto de sus grupos de interés: trabajadores, proveedores, sociedad en su conjunto, etc., pues, para este modelo, la razón fundamental de ser de toda empresa desde el punto de vista económico y social es la creación de valor actual y a largo plazo para contribuir al bienestar de todos y cada uno de sus grupos de interés (stakeholders).

El modelo resalta, entonces, la importancia de considerar en toda cadena de abastecimiento los factores duros (métodos de planeación y control, estructura organizacional, cercanía física y compartición de activos y de flujos de actividades, información y productos) y los blandos (capital social, cultura organizacional y gestión del conocimiento), de manera integrada, con énfasis en estos últimos como una estrategia clave para el óptimo funcionamiento de la cadena. La siguiente es una descripción de los tres factores blandos identificados como relevantes en la integración de la cadena de abastecimiento:

\section{Capital social}

Este factor es definido como el conjunto de redes, normas sociales y confianza que permiten la acción colectiva y determinan la cooperación y cohesión entre grupos en pos del bien común. En este sentido, de acuerdo con Putnam (2004), el capital social no es una sola entidad sino, más bien, es una variedad de entidades con dos características en común que facilitan las acciones de la interacción entre las personas y las organizaciones: la confianza y las normas sociales. Para el mencionado autor, la confianza es el aspecto más importante en todo proceso de interacción al afirmar que no es suficiente confiar en los otros, antes de interactuar y cooperar, sino que también es necesario sentir que esos otros confíen en uno.

Para los efectos de este modelo, el capital social es entendido como el conjunto de relaciones e interacciones entre los diferentes actores y los procesos derivados de estas interacciones en la cadena de abastecimiento y cuyo rol es generar y mantener excelentes relaciones entre las empresas de la red de abastecimiento para 
desarrollar transacciones positivas y sostenibles con ellas. Este capital es la base de toda la estructura relacional de la red y determina el establecimiento de las relaciones entre las empresas, el tipo de vínculo, la forma de interacción, la posición de cada empresa en la cadena, el lenguaje, las metas, los valores por compartir, los proyectos que son objeto de trabajo conjunto y el compromiso de las partes en la cadena (Villena et al., 2011). También tiene que ver con el intercambio de conocimiento que se da durante la interacción social entre las empresas de la cadena (Szeto et al., 2006; Vainio, 2005) y que ha de ser aprovechado para mejorar los retornos de esa interacción y hacer sostenible dicha cadena (Austry y Griffis, 2008; Adler y Kwon, 2002).

Este factor es muy significativo porque resalta la importancia de que cada empresa de la red conozca su rol y la necesidad de una cooperación para el logro eficaz de proyectos conjuntos y metas compartidas (Roden et al., 2014; Carey et al., 2011) desde una actitud de confianza y beneficio mutuo (Assif, 2011; Lambert y Cooper, 2000; Wu et al., 2004). Así, una cadena de abastecimiento basada en la confianza y en relaciones colaborativas, amplias y sólidas, potencia y promueve el trabajo conjunto e impacta favorablemente en el logro de los objetivos organizacionales de las empresas integrantes de la cadena. De ahí, la importancia de que las empresas se preocupen por construir sólidas relaciones de confianza mutua con los integrantes de sus cadenas de abastecimiento.

\section{Cultura organizacional}

Según Schein (2010), la cultura organizacional es un sistema de significados, valores y comportamientos compartidos, sostenido por las personas de una organización que hace que se distinga de otras organizaciones. La cultura organizacional tiene el potencial para impactar la organización, ya sea de manera positiva o negativa, y, por lo tanto, es un factor determinante de su potencial competitivo, lo cual muestra la necesidad de una sólida y funcional cultura organizacional en las empresas. Es decir, debe haber una cultura organizacional alineada con la estrategia orientada a crear identidad organizacional fundamentada en la práctica de valores humanos, caracterizada por ser flexible, que propicie la motivación, el compromiso, la autonomía para la toma de buenas decisiones, la disposición hacia el cambio, la adaptación de la organización a nuevas condiciones y desafíos del entorno y que, además, proporcione una fuerte cohesión entre sus miembros. Los beneficios de una cultura organizacional sólida, de acuerdo con Rodríguez (2009), son: valores centrales que se sostienen con intensidad y se comparten ampliamente, un fuerte vínculo de cohesión que sirve de guía para los comportamientos y el desempeño organizacional, una mejor consistencia de las decisiones por la fuerte orientación de los valores compartidos, una menor necesidad de sistemas formales y de controles por el alto compromiso logrado, identificación y mayor identidad cultural y mejor implementación de las estrategias.

Para efectos de este modelo, la cultura organizacional es entendida como el conjunto de principios compartidos entre las organizaciones de una SCM, que se puede convertir en facilitadora o innovadora de las buenas y eficaces relaciones entre los integrantes de la cadena (Inkpen et al., 2005), que está estrechamente ligada con la comunicación colaborativa y la orientación al mercado y que, por ello, requiere una comunicación empática y de confianza entre los actores para compartir metas (Agan, 2011; Connell y Voola, 2013). Según los estudios revisados, la cultura organizacional determina significativamente la disposición de las empresas a integrarse en su SCM y mientras mayores rasgos de cultura se compartan entre las organizaciones, se alcanzan mayores niveles de integración en sus SCM ((Agan, 2011; Braunscheidel et al., 2010; Connell y Voola, 2013) y mejores resultados de largo plazo para el conjunto de las empresas de la cadena. También, la cultura influye en el proceso de aprendizaje organizacional de las empresas de la cadena y, por ende, en los procesos de creación y difusión de conocimiento (Armenteros, 2012; Schein, 2010) y en la disposición de las personas para generar y compartir conocimiento (Marulanda, López y López, 2016).

\section{Gestión del conocimiento}

Es indudable que, en la sociedad actual, el conocimiento se ha convertido en un recurso clave para la competitividad de las personas, las organizaciones, las regiones y las naciones y por ello la importancia de realizar una eficaz gestión del conocimiento (GC), entendida esta como la capacidad de las personas, las organizaciones y las naciones para identificar, registrar, compartir o transferir, crear y usar conocimiento relevante para potenciar su capacidad competitiva (Bernal et al., 2016, Conell y Voola, 2013). En este sentido, la gestión del conocimiento es igualmente relevante para la eficaz gestión de las cadenas de abastecimiento porque estas son estructuras en las que se comparte, transfiere, crea y usa conocimiento inter e intraorganizacional.

En este sentido, la gestión del conocimiento entre los miembros de la red y/o cadena es visualizada como un requisito para desarrollar una filosofía de integración en la SCM, especialmente para los procesos de planeación y control. El compartir, crear y usar conocimiento es voluntad de los miembros de la cadena, pero esa voluntad es influenciada por la actitud que sobre la gestión del conocimiento se tiene por parte de las 
personas de las diferentes empresas que integran la cadena y, por ello, se presenta la necesidad de que las empresas hagan una buena gestión el conocimiento que se refleje en los resultados de la acción de esa cadena en beneficio mutuo.

Cuando en el proceso de interacción de una cadena de abastecimiento se hace una eficaz gestión del conocimiento por parte de sus diferentes actores, tales como proveedores, fabricantes, clientes, bancos, etc., se facilitan los procesos de la cadena y sus resultados mejoran hasta el punto de permitir que se generen innovaciones de diferente índole en el trabajo colaborativo. Para lograr ese propósito es importante construir relaciones de confianza, comunicación abierta, actitud colaborativa y compromiso entre los miembros de la cadena (Govindan et al., 2013; Tseng et al., 2015; Roden et al., 2014).

Con la gestión del conocimiento, la interacción entre las empresas de una cadena no se limita a una relación de carácter puramente comercial de corto plazo sino que se constituye en una relación estratégica de cooperación de largo plazo que permite identificar estrategias para superar las barreras que obstaculizan la integración de las empresas en la cadena, garantizar la construcción conjunta de capacidades dinámicas y el desarrollo de know-how distintivo, así como consolidar una cultura de aprendizaje común y continua que le facilite al conjunto y a cada una de las empresas de la cadena potenciar su capacidad competitiva mediante la creación de valor para el conjunto de sus grupos de interés.

Finalmente, se reitera que la confianza, la cooperación y el compromiso son rasgos fundamentales del capital social, de la cultura organizacional y de la gestión del conocimiento para la eficaz gestión de la cadena y lograr mantener relaciones, priorizando los resultados con visión de largo plazo entre los miembros de la red y así garantizar su sostenibilidad aportando valor agregado al conjunto de las empresas que la conforman (Anderson y Weitz, 1992). Por lo tanto, altos niveles de confianza, cooperación y compromiso en los actores de la SCM garantizan la construcción de eficaces procesos de integración capaces de generar valor para las empresas y sus grupos de interés (Aryee et al., 2008; Naslund y Hulthen, 2012).

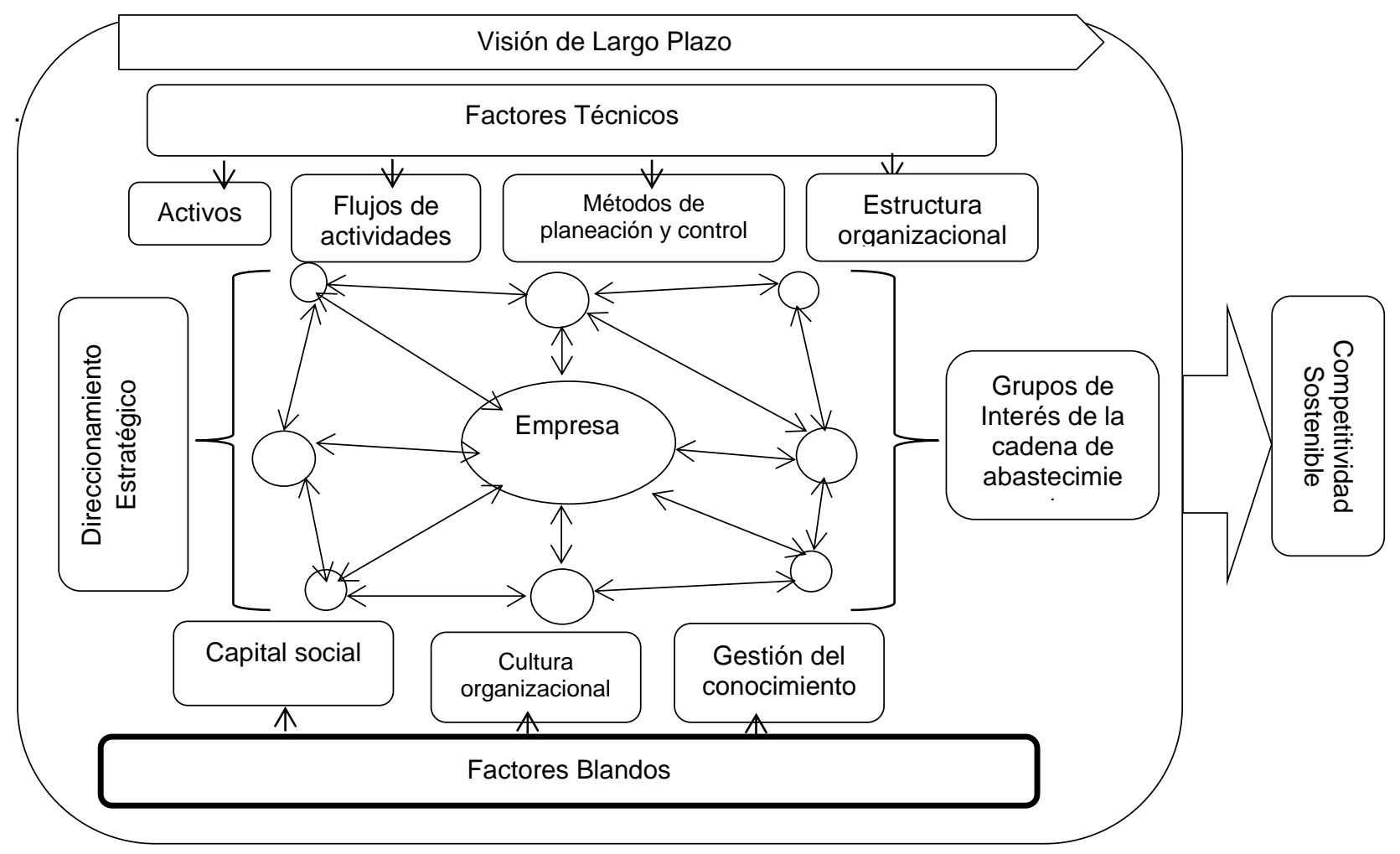

Fig. 1: Modelo de integración de la cadena de abastecimiento con énfasis en los factores blandos

La imagen central de la Figura 1 representa la cadena de abastecimiento interempresarial, el círculo central es una empresa particular y cada uno de los demás círculos a su alrededor son los otros actores que integran esa cadena (proveedores, transportadores, bancos, clientes, etc.), las líneas bidireccionales indican las interacciones y los flujos de información entre esos actores. El recuadro izquierdo representa el direccionamiento estratégico de la empresa, en donde la gestión de la cadena es una de sus estrategias organizacionales; los recuadros de la parte superior simbolizan los factores técnicos (métodos de planeación y control, estructura organizacional, cercanía física y compartición de activos y de flujos de actividades, información y productos) y los de la parte inferior indican los factores blandos (capital social, cultura 
organizacional y gestión del conocimiento), siendo estos últimos sobre los cuales se sugiere enfatizar para contribuir a la sostenibilidad de la cadena en el largo plazo cuando se les considera de forma conjunta e integral; el penúltimo recuadro de la derecha indica la creación de valor que debe generar la integración de la cadena para los grupos de interés de las empresas de esa cadena; el cuadro mayor -que encierra todo lo antes mencionado- es la integración interempresarial en la cadena que, mediante una buena gestión, debe contribuir a la construcción de la capacidad competitiva sostenible de las empresas de esa cadena (recuadro final de la derecha).

\section{DISCUSIÓN FINAL}

El modelo propuesto muestra que para una eficaz gestión de las cadenas de abastecimiento es necesario considerar tanto los factores técnicos como los blandos, siendo estos últimos altamente influyentes en esa eficacia, lo que explica el énfasis que el modelo pone en ellos. En relación con los factores blandos, el modelo muestra que hay tres factores que impactan directamente la integración de la SCM: el capital social, la cultura organizacional y la gestión del conocimiento. Mediante el capital social, las organizaciones establecen relaciones, determinan metas comunes y generan la confianza necesaria con sus socios de la cadena para desarrollar relaciones de largo plazo; con la cultura organizacional, fundamentada en valores, se consolidan esas relaciones para el logro de los intereses particulares y comunes, y mediante la gestión del conocimiento se comparte, crea y aprovecha, de manera estratégica, el conocimiento relevante que beneficia a los diferentes actores de la cadena para generar innovación y crear valor para la sostenibilidad de las empresas y la cadena.

En este sentido, la red de abastecimiento, integrada como una unidad, debe coordinarse para satisfacer de una manera más eficiente, eficaz y competitiva las necesidades actuales y futuras de las diferentes partes interesadas. Así, la integración de la SCM debe entenderse no solamente como un acuerdo entre un cliente y un proveedor sino como una interacción, en múltiples niveles, con las diferentes organizaciones con las cuales una organización establece relaciones tendientes a crear valor de manera sostenible para sí mismas y para el conjunto de sus grupos de interés.

\section{CONCLUSIONES}

De acuerdo con los antecedentes de la literatura consultada, del análisis realizado en este trabajo y en la discusión de la información y propuesta del modelo, se pueden extraer las siguientes conclusiones principales:

1.- Los estudios sobre los determinantes de la gestión de las cadenas de abastecimiento en las empresas han enfatizado más sobre los factores técnicos que sobre los factores blandos, a pesar de que se reconoce que son, básicamente, estos últimos los que más influyen en la construcción de interacciones de largo plazo en esas cadenas.

2.- Aunque la bibliografía revisada muestra que hay diversidad de factores blandos como determinantes de la gestión de la cadena de abastecimiento, esa diversidad se sintetiza en tres factores que son el capital social, la cultura organizacional y la gestión del conocimiento y los demás (confianza, colaboración, cooperación, compromiso, etc.) son rasgos de esos tres factores y no son propiamente factores.

3.- El modelo resalta la importancia de que la gestión de la cadena de abastecimiento sea considerada como una variable estratégica por parte de las empresas y que, por ende, los factores blandos determinantes de la eficacia de esas cadenas igualmente sean considerados como factores estratégicos que contribuyen a la creación de valor para las empresas y sus grupos de interés.

Finalmente, se puede decir que este estudio contribuye a la gestión del conocimiento en el tema toda vez que consolida y analiza las perspectivas de los estudios más significativos que sobre factores blandos en la integración en redes y cadenas de abastecimiento se han realizado. Sin embargo, por su carácter teórico, se requieren futuros estudios con el objeto de realizar análisis de confiabilidad y validez del modelo mediante la comprobación empírica.

\section{REFERENCIAS}

Adler, P. S. y S. W, Kwon, Social capital: Prospect for a new concept. Academy of Management Review, 27, 17-40 (2002)

Agan, Y., Impact of Operations, Marketing, and Information Technology Capabilities on Supply Chain Integration, Journal of Economic and Social Research: 13(1), 27-56 (2011)

AlSagheer, A.; H.B. Mohammed; M. Ahli; E. Airways y A. Dhabi, Impact of Supply Chain Integration on business performance and its challenges. International Business \& Economics Research Journal: 10(12), 79-93 (2011) 
Anderson, E. y B.A. Weitz, The use of the pledge to build and sustain commitment distribution channels, Marketing Scienc: 8, 310-323 (1992)

Armenteros, M.C.; L. Guerrero; F.G. Noyola y V.M. Molina, Cultura Organizacional y Organización que Aprende un Análisis desde la Perspectiva de la Innovación, Revista Internacional de Administración \& Finanzas, 5(1), (2012)

Aryee, G.; M. Naim y C. Lalwani, Supply chain integration using a maturity scale, Journal of Manufacturing Technology Management: 19(5), 559-575 (2008)

Assif, M., Supply chain commitment and business process integration, The implications of Confucian dynamism, European Journal of Marketing: 45(3), 358-382 (2011)

Austry, C. W., y S. E. Griffis, Supply Chain Capital: The Impact of Structural and Relational. Linkages on Firm Execution and Innovation. Journal of Business Logistics, 29(I), 157-173 (2008)

Bernal-Torres, C., M. Hena, C., Aguilera y S., Frost, Gestión del conocimiento y actividad empresarial en Colombia. Revista de Ciencias Sociales: XXII (1), 126-147 (2016)

Borgatti, S.P. y X. Li, On social network analysis in a supply chain Context. Journal of Supply Chain Management: 45(2), 5-22 (2009)

Braunscheidel, M. J.; N.C. Suresh y A.D. Boisnier, Investigating the Impact of Organizational Culture on Supply Chain Integration, doi: 10.1002/hrm.20381, Human Resource Management: 49(5), 883-911 (2010)

Capo-Vicedo, J.; J.V. Tomás-Miquel y M. Expósito-Landa, La Gestión del Conocimiento en la Cadena de Suministro. Análisis de la Influencia del Contexto Organizativo. Información Tecnológica: 18(1), 127-135 (2007)

Carey, S.; B. Lawson y D.R. Krause, Social capital configuration, legal bonds and performance in buyer-supplier and relationships, doi:10.1016/j.jom.2010.08.003, Journal of Operations Management: 29, 277-288 (2011)

Christopher, M., Logistics and Supply Chain Management, Pitman Publishing, London, sj_347 195 (1998)

Conell, J. y R. Voola, Knowledge integration and competitiveness: a longitudinal study of an industry cluster. Journal of Knowledge Management: 17(2), 208-225 (2013)

Dwyer, J. y H. Singh, The relational view: cooperative strategy and sources of Interorganizational competitive advantage, Academy o/ Management fleview: 23(4), 660-679 (1998)

Flynn, B. B.; B. Huo y X. Zhao, The impact of supply chain integration on performance: a contingency and configurational approach, Journal of Operations Management: 28(1), 58-71 (2010)

Fontrodona, J. y A. J. G. Sison, The Nature of the Firm, Agency Theory and Shareholder Theory: A Critique from Philosophical Anthropology, Journal of Business Ethics: 66, 33-42 (2006)

Govindan, K.; R. Khodaverdi y A. Jafarian, A fuzzy multi criteria approach for measuring sustainability performance of a supplier based on triple bottom line approach, J. of Cleaner Production: 47, 345-354 (2013)

Horn, P.; P. Sheffler y H. Schiele, Internal integration as a pre-condition for external integration in global sourcing: $A$ social capital perspective, Int. J. Production Economics: 153, 54-65 (2014)

Inkpen, A. C. y E. W. K. Tsang, Social Capital, Networks, and Knowledge Transfer. Academy of Management Review: 30(1), 146-165 (2005)

Katunzi, T.M., Obstacles to Process Integration along the Supply Chain: Manufacturing Firms Perspective, International, Journal of Business and Management: 6(5), 105-113 (2011)

Lambert D. M. y M.C. Cooper, Issues in Supply Chain Management, Industrial, Marketing Management: 29, 65-83 (2000)

Laszlo, C., The Sustainable Company: How to Create Lasting Value Through Social and Environmental Performance, Island Press, Washington, D.C., (2003)

Lawson, B.; B.B. Tyler, \& P.D. Cousins, Antecedents and consequences of social capital on buyer performance improvement, doi:10.1016/j.jom.2007.10.001, Journal of Operations Management, 26, 446-460 (2008)

Lee, P. D., Measuring Supply Chain Integration: A Social Network Approach. Supply Chain Forum An International Journal: 6(2), 58-67 (2005)

López, M.; A. Hernández y C. Marulanda, Procesos y prácticas de gestión del conocimiento en cadenas productivas de Colombia, Información Tecnológica: 25(3), 125-134 (2014)

Marulanda, C., C. López y F. López, La Cultura Organizacional y las Competencias para la Gestión del Conocimiento en las Pequeñas y Medianas Empresas (PYMEs) de Colombia, doi: 10.4067/S0718-07642016000600002, Información Tecnológica, 27(6), 3-10 (2016)

Mentzer, J. T.; W. DeWitt; J.S. Keebler; M. Soonhong; N.W. Nix; C.D. Smith y Z. Zacharia, Defining supply chain management, Journal of Business Logistics: 22(2), 1-25 (2001)

Min, S.; S.K. Kim y H. Chen, Developing Social Identity and Social Capital for Supply Chain Management. Journal Oe Business Logistics: 29(1), 283-304 (2008) 
Mohr, J. J.; R.J. Fisher y J.R. Nevin, Collaborative Communication in Interfirm Relationships: Moderating Effects of Integration and Control, Journal of Marketing: 60, 103-115 (1996)

Naslund, D. y H. Hulthen, Supply chain management integration: a critical analysis, doi: 10.1108/14635771211257963, Benchmarking: An International Journal: 19 (4/5), 481-501 (2012)

OIT, El desafío de la promoción de empresas sostenibles en América Latina y el Caribe: un análisis regional comparativo. Lima, OIT/ACTEMP, Oficina Regional para América Latina y el Caribe (2012)

Oliveira, J. F., The influence of the social capital on business performance: an analysis in the context of horizontal business networks. Revista de Administração Mackenzie: 14(3), 209-235 (2013)

Putnam, R. D., Using Social Capital to Help Integrate Planning Theory. Researeh, and Practice, Journal of the Ameritan Planning Association: 70(2), 142-151 (2004)

Roden, S. y B. Lawson, Developing social capital in buyer-supplier relationships: The contingent effect of relationshipspecific adaptations, Int. J. Production Economics: 151, 89-99 (2014)

Rodríguez, R., La cultura organizacional: un potencial activo estratégico desde la perspectiva de la administración, Invenio: 12(22), 67-92 (2009)

Romano, P., Co-ordination and integration mechanisms to manage logistics processes across supply networks, Journal of Purchasing \& Supply Management: 9, 119-134 (2003)

Sanchis, R., R. Poler y A. Ortiz, Técnicas para el Modelado de Procesos de Negocio en Cadenas de Suministro, doi:10.1612/inf.tecnol.4017it.08, Información Tecnológica: 20 (2) 29-40 (2009)

Schein, E., Organizational Culture and Leadership. 4th Ed. John Wiley \& Sons, California, United States (2010)

Simanca, M.; L. Montoya y C. Bernal, Gestión del conocimiento en la cadena láctea Colombia, Revista Información Tecnológica: 27(3). 93-106 (2016)

Son, B.G., C. Kocabasoglu-Hillmer y S. Roden, A dyadic perspective on retailer-supplier relationships through the lens of social capital, Int. J. Production Economics: 178, 120-131 (2016)

Soonhong, M. y J.T. Mentzer, Developing and measuring supply chain management concepts, Journal of Business Logistics: 25(1), 63-100 (2004)

Sukati, I.; A. Hamid; R. Baharun; M.N. Alifiad y M. Anuar, A Competitive Advantage through Supply Chain Responsiveness and Supply Chain Integration, International Journal of Business and Commerce: 1(7) (2012)

Szeto, R.; P.C. Wright y E. Cheng, Business networking in the Chinese context Its role in the formation of guanxi, social capital and ethical foundations, Management Research News: 29(7), 425-438 (2006)

Tseng, M. L.; M.K. Lim y W.P. Wong, Sustainable supply chain management: a closed-loop network hierarchical approach, Industrial Management \& Data System: 115(3), 436-461 (2015)

Turgay, T. y M.A. Ekemen, An empirical analysis on organizational integration and its effect on internationalization and exporting firms in North Cyprus, Journal of Management Research: 5(1), 359-377 doi:10.55296/jmr.v5i1.2807, (2013)

Vainio, A. M., Exchange and combination of knowledge-based resource in network relationships. A study of software firms in Finland. European Journal of Marketing, 39(9/10), 1078-1095 (2005)

Vickery, S.; J. Jayaram; C. Droge y R. Calantone, The effect of an integrative supply chain strategy on customer service and financial performance: an analysis of direct versus indirect relationship. Journal of Operations Management: 21, 52339 (2004)

Villena, V.H.; E. Revilla y T.Y. Choi, The dark side of buyer-supplier relationships: A social capital perspective. doi:10.1016/j.jom.2010.09.001, Journal of Operations Management: 29, 561-576 (2011)

Von Hippel, E., The resource of innovations, Oxford University Press, New York. NY, (1988)

Wu, W. Y.; C.Y. Chiag; Y.F. Wu y H.F. Tu, The influencing Factors of commitment and business integration on supply chain management, Industrial Management + Data Systems: 104(4): 322-333 (2004) 